\title{
Prescrição de medicamentos potencialmente inapropriados para idosos segundo os Critérios de Beers: revisão sistemática
}

\author{
Prescribing potentially inappropriate medications for the elderly \\ according to Beers Criteria: systematic review
}

Marcus Fernando da Silva Praxedes (https://orcid.org/0000-0003-4226-6926) ${ }^{1}$

Gleideson Cerqueira dos Santos Pereira (https://orcid.org/0000-0002-8793-7730) ${ }^{1}$

Claudia Feio da Maia Lima (https://orcid.org/0000-0002-4718-8683) ${ }^{1}$

Djanilson Barbosa dos Santos (https://orcid.org/0000-0002-2638-9275) ${ }^{1}$

Jamille Sampaio Berhends (https://orcid.org/0000-0002-5315-7349) ${ }^{1}$

${ }^{1}$ Centro de Ciências da Saúde, Universidade Federal do Recôncavo da Bahia. Av. Carlos Amaral 1015, Cajueiro. 44574-490 Santo Antônio de Jesus BA Brasil. marcusfera@yahoo.com.br

\begin{abstract}
The study aimed to perform a systematic review to identify and evaluate the prevalence of potentially inappropriate medicines (PIM) prescriptions for the elderly, according to Beers Criteria, in hospitalized elderly individuals aged 65 years or older. Five databases consulted: VHL; Cochrane Library; CINAHL; MEDLINE and Web of Science. Nineteen articles identified, selected based on eligibility criteria. The mean age was 78.2 years and the most used criterion for the identification of PIM for the elderly was Beers 2015 (57.9\%). A total of 221,879 elderly received a prescription for PIM, the mean prevalence was $65.0 \%$, for the gastrointestinal system (15.3\%) and proton-pump inhibitors (27.7\%) highlighted as the main class of medicine prescribed. It concluded that the Beers Criteria have made it possible to identify the high prevalence in the prescription of PIM. The results of this review may help in the decision making of health professionals, to avoid the administration of PIM and to propose best practices to ensure the safety of the elderly hospitalized.
\end{abstract}

Key words Inappropriate Prescribing, Potentially Inappropriate Medication List, Aged
Resumo Objetivou-se realizar uma revisão sistemática para identificar e avaliar a prevalência da prescrição de medicamentos potencialmente inapropriados (MPI), segundo os Critérios de Beers, em idosos hospitalizados com $\geq 65$ anos. Foram consultadas cinco bases de dados: BVS, Cochrane Library, CINAHL, MEDLINE e Web of Science. Foram identificados 19 artigos, selecionados a partir de critérios de elegibilidade. A média de idade foi 78,2 anos e o critério mais utilizado para a identificação dos MPI para idosos foi o de Beers 2015 (57,9\%). Um total de 221.879 idosos recebeu prescrição inapropriada, a prevalência média foi de $65.0 \%$, com destaque para o sistema gastrointestinal (15,3\%) e os inibidores da bomba de prótons $(27,7 \%)$ como a principal classe de medicamento prescrita. Conclui-se que os Critérios de Beers possibilitaram a identificação da alta prevalência na prescrição dos MPI. Os resultados desta revisão poderão auxiliar na tomada de decisão dos profissionais de saúde, no intuito de se evitar a administração dos MPI e propor melhores práticas que garantam a segurança do idoso hospitalizado.

Palavras-chave Prescrição Inadequada, Lista de Medicamentos Potencialmente Inapropriados, Idoso 


\section{Introdução}

A iatrogenia relacionada à terapia medicamentosa tem sido apontada pelos especialistas da área da saúde da pessoa idosa como um verdadeiro problema de saúde pública. A polifarmácia inadequada é geralmente prescrita na presença de problemas de saúde complexos e que necessitam de tratamento com um maior consumo de medicamentos ${ }^{1,2}$. Pacientes que tomam um grande número de medicamentos são mais propensos a ter prescrições potencialmente inapropriadas, contribuindo para torná-los mais vulneráveis a situações indesejáveis relacionadas à farmacoterapia, incluindo interações medicamentosas, efeitos adversos, maiores taxas de internações hospitalares e utilização de recursos de saúde $e^{3,4}$.

Neste cenário, estudos apontam explicitamente para medicamentos específicos ou categorias de medicamentos potencialmente inapropriados (MPI) que indivíduos idosos devem evitar consumir ou fazê-lo com cautela ${ }^{5,6}$. MPI são aqueles em que os riscos associados à sua utilização podem ser superiores aos benefícios terapêuticos, sobretudo quando alternativas mais eficazes estão disponíveis ${ }^{7}$. Apesar das evidências associadas com desfechos negativos, eles continuam a ser prescritos e utilizados em idosos com alta prevalência ${ }^{8-10}$. Destaca-se que os critérios são um indicador do manejo inadequado dos idosos, mas não substituem o julgamento clínico individualizado.

Em diversos países a prevalência de MPI é elevada, variando, de acordo com a ferramenta de triagem utilizada, entre $33,9 \%$ e $58 \%$ no contexto domiciliar e entre $42,4 \%$ e $60,5 \%$ em pacientes hospitalizados ${ }^{11}$. No âmbito nacional, a prevalência também é alta, chegando a 59,2\% em contexto domiciliar ${ }^{12}$ e a $85,9 \%$ em hospitais ${ }^{13}$. O uso de MPI no contexto hospitalar está associado a vários desfechos desfavoráveis, dentre eles, o aumento do tempo de internação e maior mortalidade desta população $0^{14,15}$. Nesse cenário, a realização de estudos que avaliem a prevalência de MPI em idosos hospitalizados é fundamental, devido à sua maior vulnerabilidade nesta situação.

Existem diferentes ferramentas de triagem para identificar e avaliar a prevalência de MPI em pacientes idosos que estabelecem critérios explícitos de avaliação. Destacam-se a Screening Tool of Older Persons' Prescriptions (STOPP) ${ }^{6}$ e os Critérios de Beers ${ }^{5,16}$. Esses últimos são os mais utilizados no mundo e se tornaram uma ferramenta útil para avaliar a qualidade da prescrição para idosos, incluindo avaliações geriátricas es- pecíficas em relação ao uso de medicamentos. Esses critérios buscam descrever os medicamentos a serem evitados por idosos e foram elaborados por Beers et al. ${ }^{16}$, em 1991. Foram expandidos e revisados mais recentemente em $2012^{17}, 2015^{18}$ e $2019^{5}$, pela Sociedade Americana de Geriatria para serem aplicados a todas as pessoas com idade $\geq 65$ anos, seguindo uma abordagem baseada em evidências, exceto para idosos em cuidados paliativos. O uso de uma ferramenta amplamente utilizada no mundo permite um conhecimento mais amplo da real situação do uso de medicamentos inapropriados por idosos e permite a comparação de estudos realizados em diferentes regiões geográficas.

Indícios científicos, baseados em estudos realizados por meio de métodos padronizados ${ }^{19} \mathrm{e}$ que utilizem critérios explícitos para avaliação da terapia medicamentosa do idoso, são fundamentais para se melhorar a seleção de medicamentos; educar profissionais de saúde e pacientes; reduzir eventos adversos; e servir como uma ferramenta para avaliar a qualidade dos cuidados, custos e padrões de uso de medicações em adultos mais velhos $^{18}$. Não foi identificada, na literatura atual, nenhuma revisão sistemática sobre o uso de MPI na população idosa hospitalizada que utilizasse os critérios de Beers. Nesse sentido, o objetivo desse estudo foi realizar uma revisão sistemática para identificar e avaliar a prevalência da prescrição de MPI, segundo os Critérios de Beers, em idosos hospitalizados com $\geq 65$ anos.

\section{Método}

Um protocolo específico foi desenhado pelos pesquisadores para estruturação da revisão sistemática e registrado no PROSPERO, disponível em: https://www.crd.york.ac.uk/prospero/display_ record.php?ID=CRD42019133367. Constaram no referido protocolo as etapas de definição do tema e elaboração da questão de pesquisa, "Qual a prevalência da prescrição de MPI, segundo os Critérios de Beers, em idosos hospitalizados com $\geq 65$ anos?"; elaboração dos critérios de elegibilidade, inclusão e exclusão dos estudos; levantamento das publicações nas bases de dados; categorização e análise das informações encontradas nas publicações e avaliação descritiva dos estudos selecionados.

A seguinte combinação de descritores (MeSH) em inglês foi usada para conduzir a busca na base de dados MEDLINE, assim como nas demais bases de dados, com pequenas adaptações, 
de acordo com as suas especificidades: ("Critérios de Beers") AND ("Prescrição Inapropriada”) $A N D$ ("Lista de medicamentos potencialmente inapropriados") e ("Potentially Inappropriate Medication List") AND ("Inappropriate Prescribing") AND ("Beers Criteria" OR "Beers Criteria, de" OR “ Beers Potentially Inappropriate Medications"), com os limites de data [01/02/2012 a 31/05/2019]. Um bibliotecário foi consultado para promover rigor no processo de busca. Foram utilizadas cinco bases de dados: Biblioteca Virtual em Saúde do Ministério da Saúde (BVS/ MS); Cochrane Library; Cumulative Index to Nursing \& Allied Health Literature (CINAHL); Medical Literature Analysis and Retrieval System Online (MEDLINE) e Web of Science. A busca dos estudos foi realizada no mês de maio de 2019.

Os critérios de elegibilidade foram estudos observacionais e experimentais, realizados entre 2012 e 2019; pacientes com $\geq 65$ anos internados em hospitais; estudos que analisaram as prescrições de medicamentos usando os Critérios de Beers nas versões de 2012 ou 2015, sem restrição de idioma. Foram excluídos os resumos de artigos de conferências, teses e dissertações e estudos que incluíssem análise conjunta de dados com mais de um critério para a avaliação de MPI. A atualização de 2019 dos Critérios de Beers não foi incluída devido a sua recente publicação e a falta de estudos publicados com sua aplicação.

Os estudos foram selecionados seguindo dois passos. Primeiramente, dois revisores treinados (R1 e R2) fizeram a leitura e avaliação independente dos títulos e resumos dos artigos identificados nas bases de dados eletrônicas. De acordo com os critérios de elegibilidade, selecionaram os artigos para leitura completa. Posteriormente, foi feita a leitura na íntegra e inclusão final dos artigos. Quando não houve consenso entre os revisores sobre a inclusão e seleção final dos estudos, foi considerada a opinião de um terceiro revisor (R3). A qualidade metodológica dos estudos observacionais foi analisada através da Escala de Newcastle-Ottawa, que pontua três componentes: seleção dos grupos ( $0-4$ pontos), qualidade de ajuste para confusão (0-2 pontos) e avaliação da exposição após desfecho (0-3 pontos). A qualidade satisfatória dessa escala deve fornecer pontuação $\geq 6$, em que a pontuação de 9 pontos representa alta qualidade metodológica ${ }^{20}$.

Para sistematização da extração dos dados foi utilizado um formulário específico contendo: título, periódico, autor, ano, país, tipo de publicação; desenho do estudo: tipo de estudo, objetivos, método de coleta e amostragem de dados, crité- rios de elegibilidade, uso de instrumentos; participantes: número de participantes do estudo; identificação de MPI para idosos de acordo com os critérios da Beers (versões de 2012 ou 2015); limitações: risco de viés. O processo de busca e seleção dos estudos seguiu as recomendações PRISMA $^{21}$ e está representado na Figura 1.

As evidências das prescrições de MPI foram identificadas, exploradas e sintetizadas de forma narrativa com a tabulação dos resultados dos estudos incluídos. Alguns não identificaram a prevalência das prescrições que continham MPI, impossibilitando o cálculo e a comparação direta dos resultados, portanto, a realização de uma meta-análise não seria recomendada. Destaca-se também que as versões 2012 e 2015 dos critérios da Beers apresentam diferenças quanto às categorias de MPI, o que dificulta a análise conjunta dos dados. Nesse sentido, a prevalência de MPI foi analisada de forma geral, independente da inclusão do medicamento em determinada categoria. Assim, os resultados foram conduzidos, a partir da análise descritiva e sintetizados, de acordo com a prevalência dos MPI.

\section{Resultados}

O processo de busca resultou na identificação de 721 estudos e após as etapas de seleção 19 estudos observacionais foram incluídos (Figura 1).

Dentre os estudos selecionados, 17 (89,5\%) eram estudos observacionais de coorte retrospectivo, publicados de 2013 a 2019. A China destacou-se com o maior número de publicações $(\mathrm{n}=4 ; 21,0 \%)$. O tamanho amostral variou de 104 a 313.733 pacientes e a duração dos estudos de três a trinta e cinco meses. A média de idade foi 78,2 anos $(71,5-84,8)$. O critério mais utilizado para a identificação dos MPI para idosos foi o de Beers 2015 ( $n=11 ; 57,9 \%)$. Todos os estudos apresentaram boa qualidade metodológica de acordo com a escala de Newcastle-Ottawa. As características gerais dos estudos estão sumarizadas na Tabela 1 .

A prevalência média de MPI foi de $65,0 \%$ (28,7-95,3\%), em que 221.879 idosos fizeram uso de medicamentos inapropriados. Destacouse a prescrição para o sistema gastrointestinal $(15,3 \%)$, medicamentos para dor $(10,5 \%)$ e sistema nervoso central $(9,7 \%)$ (Tabela 1). Os principais MPI prescritos foram inibidores da bomba de prótons - IBP $(27,7 \%)$, opioides $(27,2 \%)$ e benzodiazepínicos (19,0\%) (Tabela 2). 


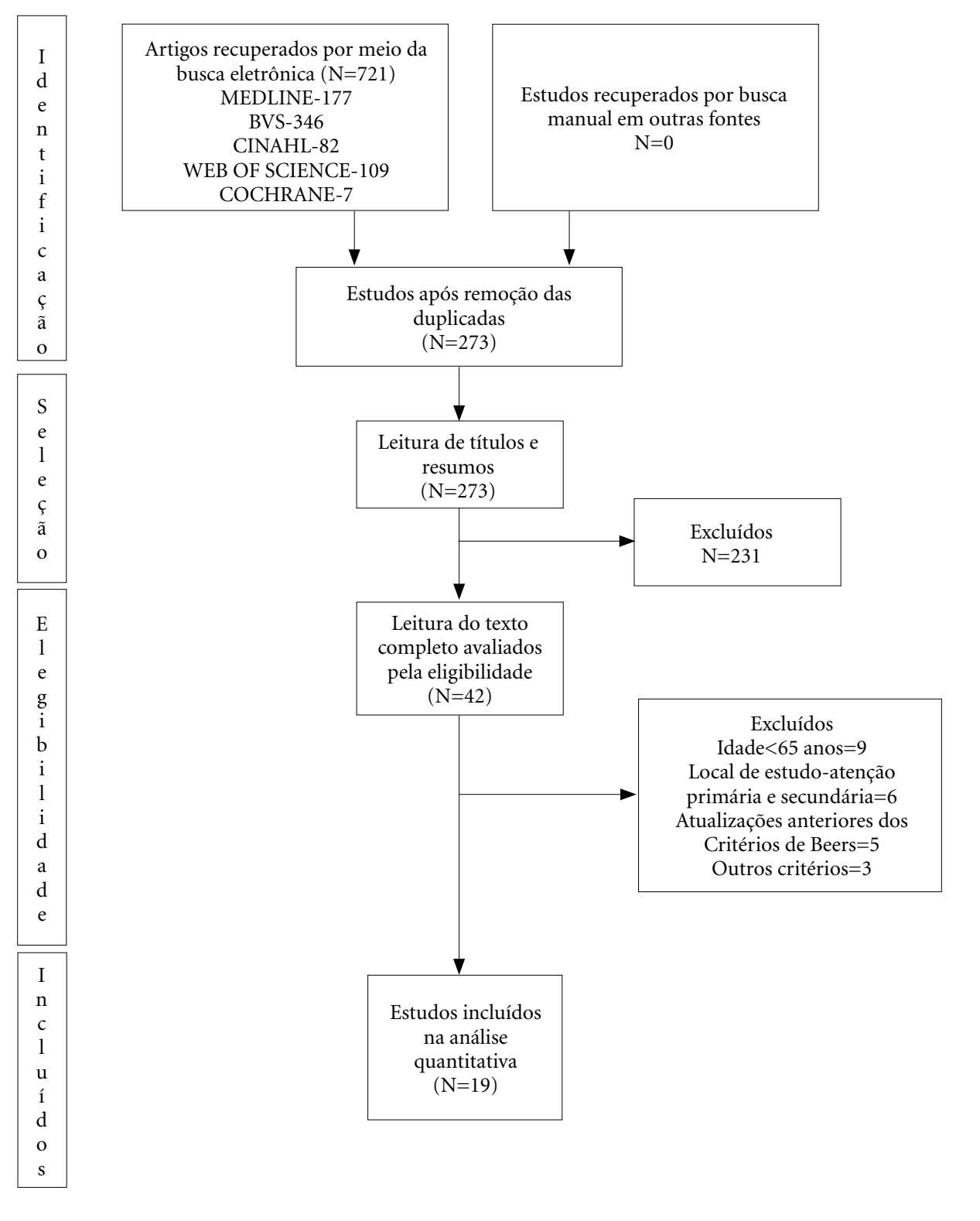

Figura 1. Fluxograma de seleção dos estudos.

Fonte: Elaborado pelos autores, adaptado do Preferred Reporting Items for Systematic Review and Meta-Analyses (PRISMA).

\section{Discussão}

A presente revisão sistemática possibilitou a determinação da prevalência de MPI prescritos para idosos hospitalizados. A identificação desses medicamentos se mostra relevante e possibilita o conhecimento atual da terapia medicamentosa nessa população específica. A alta prevalência de MPI observada $(65,0 \%)$ é preocupante, indi- cando a importância de intervenções para a sua redução. Estudos realizados no Canadá ${ }^{23}$ e nos $\mathrm{EUA}^{30}$, apontaram taxas superiores a $90.0 \%$. No Brasil, Japão e Itália identificou-se uma prevalência de $47,3 \% \%^{41}, 56,1 \%{ }^{42}$ e $31,1 \%{ }^{40}$, respectivamente. A comparação dos resultados dos estudos realizados em todo o mundo não é simples, devido às variações nos métodos utilizados. Assim, observa-se a diferença na prevalência dessas pres- 
Tabela 1. Características dos estudos incluídos na revisão sistemática.

\begin{tabular}{|c|c|c|c|c|c|c|c|}
\hline Estudo/Ano & País & $\begin{array}{l}\text { Tipo de } \\
\text { estudo }\end{array}$ & $\begin{array}{c}\text { Amostra } \\
\mathbf{n}\end{array}$ & $\begin{array}{c}\text { Média de } \\
\text { idade (anos) }\end{array}$ & $\begin{array}{l}\text { Critérios } \\
\text { de Beers }\end{array}$ & $\begin{array}{c}\text { Prevalência } \\
\text { MPI/Paciente } \\
\text { N (\%) }\end{array}$ & $\begin{array}{l}\text { Escore de } \\
\text { qualidade }\end{array}$ \\
\hline $\begin{array}{l}\text { Chukwulebe et } \\
\text { al., } 2019^{22}\end{array}$ & $\begin{array}{l}\text { Estados } \\
\text { Unidos }\end{array}$ & Coorte & 11822 & $\begin{array}{c}71,5 \\
(67,0-78,0)\end{array}$ & 2015 & $3392(28,7)$ & 6 \\
\hline $\begin{array}{l}\text { Lester et al., } \\
2019^{23}\end{array}$ & Canadá & Transversal & 319 & $\begin{array}{c}76.0 \\
(75,0-76,8)\end{array}$ & 2015 & $304(95,3)$ & 6 \\
\hline Ma et al., $2019^{24}$ & China & Transversal & 863 & $\begin{array}{c}75,4 \\
(65,0-98,0)\end{array}$ & 2015 & $501(58,1)$ & 6 \\
\hline $\begin{array}{l}\text { Chang et al., } \\
2018^{25}\end{array}$ & Taiwan & Transversal & 313.733 & 76,7 & 2015 & $199.882(63,7)$ & 7 \\
\hline $\begin{array}{l}\text { Komagamine, } \\
2018^{26}\end{array}$ & Japão & Transversal & 689 & $\begin{array}{c}82,0 \\
(76,0-88,0)\end{array}$ & 2015 & $330(47,9)$ & 6 \\
\hline $\begin{array}{l}\text { Komagamine et } \\
\text { al., } 2018^{27}\end{array}$ & Japão & Transversal & 136 & 81,1 & 2015 & $105(77,2)$ & 6 \\
\hline $\begin{array}{l}\text { Najjar et al., } \\
2018^{28}\end{array}$ & $\begin{array}{l}\text { Arábia } \\
\text { Saudita }\end{array}$ & Coorte & 200 & 78,0 & 2015 & $122(61,0)$ & 7 \\
\hline $\begin{array}{l}\text { Sarwar et al., } \\
2018^{29}\end{array}$ & Paquistão & Transversal & 385 & $\begin{array}{c}78 \\
(65,0-85,0)\end{array}$ & 2015 & $342(88,8)$ & 6 \\
\hline $\begin{array}{l}\text { Sharma et al., } \\
2018^{30}\end{array}$ & $\begin{array}{l}\text { Estados } \\
\text { Unidos }\end{array}$ & Transversal & 104 & $82(70,0-90,0)$ & 2015 & $104(91,3)$ & 6 \\
\hline $\begin{array}{l}\text { Komagamine e } \\
\text { Hagane, } 2017^{31}\end{array}$ & Japão & Coorte & 158 & 84,8 & 2015 & $124(78,4)$ & 6 \\
\hline Li et al., $2017^{32}$ & China & Transversal & 6337 & 81,3 & 2012 & $4593(72,5)$ & 7 \\
\hline $\begin{array}{l}\text { Zhang et al., } \\
2017^{33}\end{array}$ & China & Transversal & 456 & $\begin{array}{c}81,8 \\
(65,0-103,0)\end{array}$ & 2015 & $244(53,5)$ & 6 \\
\hline $\begin{array}{l}\text { Arellano et al., } \\
2016^{34}\end{array}$ & Chile & Coorte & 250 & $\begin{array}{c}77,5 \\
(65,0-97,0)\end{array}$ & 2012 & $198(79,2)$ & 6 \\
\hline $\begin{array}{l}\text { Di Giorgio et } \\
\text { al.,2016 }\end{array}$ & Itália & Coorte & 1027 & 73,3 & 2012 & $507(49,0)$ & 7 \\
\hline $\begin{array}{l}\text { Rongen et al., } \\
2016^{36}\end{array}$ & Holanda & Transversal & 164 & $\begin{array}{c}74,9 \\
(65,0-92,0)\end{array}$ & 2012 & $77(47,0)$ & 6 \\
\hline $\begin{array}{l}\text { Yang et al., } \\
2015^{37}\end{array}$ & China & Transversal & 141 & $\begin{array}{c}81,5 \\
(65,0-97,0)\end{array}$ & 2012 & $94(66,7)$ & 6 \\
\hline $\begin{array}{l}\text { Matanovic } \\
\text { e Vlahovic- } \\
\text { Palcevski, 2014 }\end{array}$ & Croácia & Coorte & 454 & 74,0 & 2012 & $263(57,9)$ & 6 \\
\hline $\begin{array}{l}\text { Tosato et al., } \\
2014^{39}\end{array}$ & Itália & Coorte & 871 & 80,2 & 2012 & $509(58,4)$ & 7 \\
\hline $\begin{array}{l}\text { Napolitano et } \\
\text { al., } 2013^{40}\end{array}$ & Itália & Transversal & 605 & 76,7 & 2012 & $188(31,1)$ & 6 \\
\hline
\end{tabular}

crições, que pode estar também relacionada à organização dos sistemas de prestação de cuidados de saúde e com ambientes de prática clínica específicos em cada país ${ }^{40}$.

Incluídos na atualização de 2015 dos Critérios de Beers ${ }^{18}$, os IBP foram os principais MPI prescritos. Tais medicamentos são importantes para o tratamento de doenças relacionadas ao ácido gástrico, como doenças do refluxo gastroesofágico e úlcera péptica ${ }^{24}$. No entanto, o seu uso superior a oito semanas não é recomendado, exceto em circunstâncias específicas, quando a terapia de supressão ácida de longo prazo é indi$\mathrm{cada}^{18}$. O uso prolongado de IBP compromete a segurança do idoso e pode causar, dentre outros problemas, fraturas ${ }^{43}$, diarreia associada ao Clostridium difficile ${ }^{44}$ e risco aumentado de pneumonia ${ }^{45}$. Quando for necessário o uso prolongado, oportunidades de suspensão ou diminuição de doses devem ser consideradas. 
Tabela 2. Prevalência dos medicamentos potencialmente inapropriados para idosos, segundo Critérios de Beers da Sociedade Americana de Geriatria (2012 e 2015).

\begin{tabular}{|c|c|c|c|}
\hline $\begin{array}{l}\text { Sistema de Órgãos, } \\
\text { Categoria Terapêutica }\end{array}$ & $\begin{array}{l}\text { Prevalência } \\
\text { média (\%) }\end{array}$ & Medicamento (\%) & $\begin{array}{l}\text { Prevalência } \\
\text { média (\%) }\end{array}$ \\
\hline \multirow[t]{4}{*}{ Anticolinérgicos } & 5,7 & Difenidramina & 7,9 \\
\hline & & Anticolinérgicos & 7,2 \\
\hline & & Anti-histamínicos de primeira geração & 6,9 \\
\hline & & Antiespasmódicos & 6,7 \\
\hline \multirow[t]{2}{*}{ Antitrombóticos } & 5,1 & Ticlopina & 6,3 \\
\hline & & Antitrombóticos & 3,9 \\
\hline \multirow[t]{2}{*}{ Anti-infecciosa } & 2,8 & Anti-infecciosa & 5,1 \\
\hline & & Nitrofurantoína & 0,5 \\
\hline \multirow[t]{12}{*}{ Cardiovascular } & 8,1 & Medicamentos cardíacos & 27,0 \\
\hline & & Amiodarona & 12,4 \\
\hline & & Espironolactona & 12,1 \\
\hline & & Antiarrítmicos & 11,1 \\
\hline & & Doxazosina & 7,6 \\
\hline & & Clonidina & 7,4 \\
\hline & & Terazosina & 5,3 \\
\hline & & Bloqueadores alfa1 & 4,0 \\
\hline & & Nifedipina & 4,3 \\
\hline & & Digoxina & 3,2 \\
\hline & & Verapamil & 2,0 \\
\hline & & Propafenona & 1,1 \\
\hline \multirow[t]{15}{*}{ Sistema nervoso central } & 9,7 & Medicamentos do sistema nervoso central & 33,8 \\
\hline & & Benzodiazepínicos & 19,0 \\
\hline & & TCA terciários & 16,5 \\
\hline & & Antipsicóticos & 16,4 \\
\hline & & Hipnóticos (não benzodiazepínicos) & 14,0 \\
\hline & & Antidepressivos & 11,7 \\
\hline & & Inibidores Seletivos da Recaptação de Serotonina & 6,6 \\
\hline & & Barbitúricos & 6,5 \\
\hline & & Medicamentos anticonvulsivos & 4,6 \\
\hline & & Diazepam & 4,3 \\
\hline & & Promazina & 4,2 \\
\hline & & Lorazepam & 3,9 \\
\hline & & Haloperidol & 1,6 \\
\hline & & Olanzapina & 1,5 \\
\hline & & Meprobamato & 1,0 \\
\hline \multirow[t]{4}{*}{ Endócrino } & 7,8 & Insulina & 14,0 \\
\hline & & Sulfoniluréias de longa duração & 8,6 \\
\hline & & Megestrol & 5,27 \\
\hline & & Medicamentos endócrinos & 3,4 \\
\hline \multirow[t]{5}{*}{ Gastrointestinal } & 15,3 & Medicamentos gastrintestinais & 28,9 \\
\hline & & IBP & 27,7 \\
\hline & & Metoclopramida & 12,7 \\
\hline & & Ranitidina & 6,3 \\
\hline & & Óleo de vaselina & 1,0 \\
\hline \multirow[t]{5}{*}{ Medicamentos para a dor } & 10,5 & Opioides & 27,2 \\
\hline & & $\operatorname{AINE}(0,4-87,7 \%)$ & 15,9 \\
\hline & & Meperidina & 5,5 \\
\hline & & Medicamentos para dor & 3,4 \\
\hline & & Relaxante muscular esquelético & 0,6 \\
\hline
\end{tabular}

Notas: TCA: antidepressivo tricíclico, IBP: inibidor da bomba de prótons, AINE: anti-inflamatório não esteroidais. 
Destacaram-se também as prescrições de medicamentos opioides e anti-inflamatório não esteroidais (AINE). Os opioides foram adicionados à lista de medicamentos do sistema nervoso central (SNC) que devem ser evitados em idosos devido a sua associação com o risco de quedas ou fraturas ${ }^{46}$. Em estudo realizado, pacientes em uso de opioides tiveram 2,4 vezes mais chances de ter uma queda e maior risco de morte intra -hospitalar (RC=1,58; IC95\%=1,34-1,86) após o evento ${ }^{47}$. Os AINE são comumente usados pelos idosos para a dor musculoesquelética crônica ${ }^{48}$, entretanto, há um alto risco para a toxicidade gastrointestinal. Os idosos em uso de AINE estão em maior risco de complicações graves, por exemplo, hemorragia ou perfuração gástrica ${ }^{49}$. Recentemente, o uso de AINE tem sido relacionado a efeitos colaterais cardiovasculares ${ }^{50}$. Recomenda-se, então, o uso desses medicamentos em doses mínimas efetivas pelo menor tempo possível $^{51}$.

Os benzodiazepínicos também foram frequentemente prescritos. Com o envelhecimento, mais idosos sofrem com insônia e depressão, fazendo com que tais medicamentos sejam prescritos com maior frequência. Estudos demonstram que o uso desses medicamentos aumenta a incidência de quedas, fraturas de quadril, estado mental alterado e delirium em idosos ${ }^{52}$ e está associada a uma frequência 3,6\% maior de consultas de retorno ao departamento de emergên$\mathrm{cia}^{22}$. Nesse contexto, tem sido sugerida a terapia não farmacológica como o método inicial no tratamento de insônia ou delirium ${ }^{32}$. Quando a desprescrição do medicamento não for possível, torna-se fundamental o acompanhamento da eficácia e das possíveis RAM.

Em relação a isso, tem-se que o uso de medicamentos incluídos nos Critérios de Beers pode ser necessário em determinados momentos. Tais critérios foram criados não apenas para identificar MPI, mas também para embasar a discussão sobre os motivos pelos quais esses medicamentos foram prescritos e as situações em que seu uso pode trazer maior ou menor risco ao idoso. Portanto, os critérios visam apoiar, em vez de ter domínio sobre um bom julgamento clínico ${ }^{53}$.

Discussões interessantes foram acrescentadas pelos estudos analisados. A polifarmácia foi associada a um risco aumentado para o uso de $\mathrm{MPI}^{38}$, que pode levar a maior ocorrência de RAM e consequentemente ao aumento do tempo de internação e custos mais elevados aos sistemas de saúde $\mathrm{d}^{54,55}$. Tosato et al. ${ }^{39}$ observaram que o uso de MPI estava associado a RAM ou declínio na função física $(\mathrm{RC}=1,74$; IC95\%=1,06-2,85). Lester et al..$^{23}$ identificaram que o número de MPI prescritos não foi alterado durante a internação do paciente e o uso dos mesmos foi associado a uma taxa de incidência de 1,46 (IC95\%=1,13 a 1.88) para a duração da estadia. Os autores discorrem que possivelmente não há realização de revisões efetivas de medicação ou que os prescritores desconhecem os Critérios de Beers e a existência de medicamentos mais apropriados para uso em idosos ${ }^{23}$.

Diante de tal situação, intervenções educativas para conscientizar os prescritores sobre esses critérios se mostram relevantes. Estudos demonstraram que há uma diminuição de MPI prescritos após processos educacionais. Najjar et al. ${ }^{28}$ demostraram que houve uma diminuição significativa na taxa de incidência de MPI de $61 \%$ para 29,5\% durante a internação hospitalar $(\mathrm{p}<0,001)$; Komagamine e Hagane $\mathrm{e}^{31}$ apontaram que a proporção de pacientes que tomaram qualquer MPI diminuiu significativamente de $93,5 \%$ na admissão para $51,6 \%$ na alta $(\mathrm{p}<0,001)$. Ressalta-se que, as intervenções descritas não podem confirmar os benefícios clínicos obtidos, uma vez que não foram avaliadas usando resultados clinicamente relevantes, como mortalidade e qualidade de vida. Entretanto, as intervenções descritas podem melhorar a prescrição e aumentar a segurança no uso de medicamentos. Tais resultados afirmam que os Critérios de Beers se destacam como uma importante ferramenta para a detecção de MPI e norteiam a mudança do padrão das prescrições, a fim de se garantir uma maior segurança no uso dos medicamentos.

Outras importantes iniciativas para a redução do uso de MPI são a revisão da prescrição com desprescrição medicamentosa e sistemas informatizados ${ }^{23,31}$. A desprescrição visa à retirada planejada e gradual dos MPI para cada paciente e requer a monitorização de eventos adversos ou sintomas de rebote ${ }^{56,57}$. Tal atitude se mostra efetiva na diminuição da polifarmácia inadequada e redução de danos ao paciente ${ }^{57}$. Destaca-se também a tomada de decisão baseada em ferramentas informatizadas. Por meio do meio digital, os profissionais prescritores podem acessar todas as informações sobre a terapia medicamentosa dos pacientes e no momento do preenchimento da prescrição, alertas de risco e informações sobre interações medicamentosas são emitidos ${ }^{2,53,58,59}$. Apesar dos resultados positivos, todos os estudos deixam claro que, para a manutenção do sucesso das intervenções, é fundamental o envolvimento e aceitação do prescritor em mudar sua prática 
e a participação de uma equipe multidisciplinar, em específico enfermeiros, farmacêuticos clínicos e médicos. Assim, a garantia do objetivo comum, que é a melhoraria na qualidade da prescrição e o uso racional de medicamentos na população idosa, poderá ser alcançada.

Ao interpretar os resultados dos artigos incluídos, vale a pena mencionar algumas limitações ao método que poderiam influenciar nas estimativas. A maioria dos estudos foi do tipo retrospectivo e em um único hospital. O uso de somente uma ferramenta de identificação dos MPI - Critérios de Beers - pode ter subestimado a frequência de inadequação, uma vez que alguns medicamentos, especialmente aqueles que não são usados nos Estados Unidos, não estão incluídos.

Destaca-se também a atualização dos critérios de Beers em 2012 e 2015, gerando duas versões diferentes. A primeira com três categorias: medicamentos ou classes de medicamentos inapropriados, independentemente do diagnóstico, seus potenciais riscos e algumas de suas dosagens; medicamentos que devem ser evitados em situações clínicas específicas; e medicamentos ou classes de medicamentos que devem ser usados com cautela. A versão de 2015 , além das três categorias supracitadas, apresenta uma quarta, referente a medicamentos cujas doses devem ser ajustadas, a depender do clearance de creatinina do paciente, e uma quinta lista, com medicamentos que devem ser evitados em associação, devido a interações medicamento-medicamento. Dessa forma, houve a exclusão e inclusão de medicamentos na versão de $2015^{18}$, o que pode ter interferido na prevalência de MPI encontrada nesse estudo. Devido à falta de estudos utilizando a versão de 2019, a mesma não foi avaliada nessa revisão, o que pode ter comprometido na identificação atual da prevalência de MPI. No entanto, mesmo com essas limitações, acredita-se não haver prejuízo para os resultados e as conclusões.

\section{Conclusões}

As evidências encontradas nos estudos mostram que há uma alta prevalência de MPI prescritos. Os Critérios de Beers possibilitaram a identificação dos medicamentos que devem ser evitados em idosos, possuem critérios explícitos e o uso dos mesmos pode auxiliar na prevenção dos eventos adversos decorrentes da terapia medicamentosa inadequada. Os profissionais de saúde poderão utilizar os resultados desta revisão para a tomada de decisão, no intuito de se evitar a administração dos MPI e propor melhores práticas que garantam a segurança do idoso hospitalizado.

O pequeno número de pesquisas identificado pela revisão sistemática em idosos com $\geq 65$ anos em ambiente hospitalar, chama a atenção para a necessidade de realização de mais estudos de coorte e ensaios clínicos randomizados que utilizem os critérios de Beers como medida de qualidade do cuidado hospitalar. Somente assim, será comprovada a validade preditiva desses critérios nesse cenário. Entretanto, tais pesquisas devem seguir métodos padronizados, a fim de validar o uso dos critérios nos países de sua aplicação, possibilitando assim que os resultados dos diferentes estudos sejam mais comparáveis entre si. 


\section{Colaboradores}

CFM Lima e MFS Praxedes foram responsáveis pela concepção, pesquisa, metodologia e redação final do artigo. GCS Pereira, JS Berhends e DB Santos foram responsáveis pela metodologia e revisão crítica.

\section{Referências}

1. Gnjidic D, Le Couteur DG, Pearson S-A, McLachlan AJ, Viney R, Hilmer SN, Blyth FM, Joshy G, Banks E. High risk prescribing in older adults: prevalence, clinical and economic implications and potential for intervention at the population level. BMC Public Health 2013; 13(1):115.

2. Lai X, Zhu H, Huo X, Li Z. Polypharmacy in the oldest old ( $>/=80$ years of age) patients in China: a crosssectional study. BMC Geriatrics 2018; 18(1):64.

3. Hanlon JT, Perera S, Newman AB, Thorpe JM, Donohue JM, Simonsick EM, Shorr RI, Bauer DC, Marcum ZA, Health ABC Study. Potential drug-drug and drug-disease interactions in well-functioning community-dwelling older adults. J Clin Pharm Ther 2017; 42(2):228-233.

4. Hagstrom K, Nailor M, Lindberg M, Hobbs L, Sobieraj DM. Association between potentially inappropriate medication use in elderly adults and hospital-related outcomes. J Am Geriatr Soc 2015; 63(1):185-186.

5. 2019 American Geriatrics Society Beers Criteria ${ }^{\circledR}$ Update Expert Panel. American Geriatrics Society 2019 Updated AGS Beers Criteria ${ }^{\circledR}$ for Potentially Inappropriate Medication Use in Older Adults. J Am Geriatr Soc 2019; 67(4):674-694.

6. O'Mahony D, O'Sullivan D, Byrne S, O'Connor MN, Ryan C, Gallagher P. STOPP/START criteria for potentially inappropriate prescribing in older people: version 2. Age Ageing 2015; 44(2):213-238.

7. Renom-Guiteras A, Meyer G, Thurmann PA. The EU(7)-PIM list: a list of potentially inappropriate medications for older people consented by experts from seven European countries. Eur J Clin Pharmacol 2015; 71(7):861-875.

8. Saboor M, Kamrani AA, Momtaz YA, Sahaf R. Prevalence and associated factors of potentially inappropriate medications among Iranian older adults. Med Glas (Zenica) 2019; 16(1):121-127.

9. Mazhar F, Akram S, Malhi SM, Haider N. A prevalence study of potentially inappropriate medications use in hospitalized Pakistani elderly. Aging Clin Exp Res 2018; 30(1):53-60.

10. Lopes LM, Figueiredo TP, Costa SC, Reis AMM. Utilização de medicamentos potencialmente inapropriados por idosos em domicílio. Cien Saude Colet 2016; 21(11):3429-3438.

11. Thomas RE, Thomas BC. A Systematic Review of Studies of the STOPP/START 2015 and American Geriatric Society Beers 2015 Criteria in Patients $\geq 65$ Years. Curr Aging Sci 2019; 12(2):121-154.

12. Baldoni AO, Ayres LR, Martinez EZ, Dewulf NLS, Santos V, Pereira LRL. Factors associated with potentially inappropriate medications use by the elderly according to Beers criteria 2003 and 2012. Int J Clin Pharm 2014; 36(2):316-324.

13. Machado LPB. Avaliação do uso de medicamentos inapropriados por idosos, segundo o critério de Beers, em um hospital terciário do Distrito Federal [monografia]. Ceilândia: Universidade de Brasília; 2015.

14. Danisha P, Dilip C, Mohan PL, Shinu C, Parambil JC, Sajid M. Identification and evaluation of potentially inappropriate medications (PIMs) in hospitalized geriatric patients using Beers criteria. J Basic Clin Physiol Pharmacol 2015; 26(4):403-410. 
15. Heider D, Matschinger H, Meid AD, Quinzler R, Adler J-B, Günster C, Haefeli WE, König H-H. Health Service Use, Costs, and Adverse Events Associated with Potentially Inappropriate Medication in Old Age in Germany: Retrospective Matched Cohort Study. Drugs Aging 2017; 34(4):289-301.

16. Beers MH, Ouslander JG, Rollingher I, Reuben DB, Brooks J, Beck JC. Explicit criteria for determining inappropriate medication use in nursing home residents. UCLA Division of Geriatric Medicine. Arch Intern Med 1991; 151(9):1825-1832.

17. American Geriatrics Society 2012 Beers Criteria Update Expert Panel. American Geriatrics Society updated Beers Criteria for potentially inappropriate medication use in older adults. J Am Geriatr Soc 2012; 60(4):616-631.

18. American Geriatrics Society 2015 Beers Criteria Update Expert Panel. American Geriatrics Society 2015 Updated Beers Criteria for Potentially Inappropriate Medication Use in Older Adults. J Am Geriatr Soc 2015; 63(11):2227-2246.

19. Praxedes M, Abreu M, Ribeiro DD, Marcolino MS, Paiva SM, Martins MAP. Cross-cultural Adaptation of the Oral Anticoagulation Knowledge Test to the Brazilian Portuguese. Cien Saude Colet 2017; 22(5):16151629.

20. Wells GA, Shea B, O'Connell D, Peterson J, Welch V, Losos M, Tugwell P. The Newcastle-Ottawa Scale (NOS) for assessing the quality of nonrandomised studies in meta-analyses [Internet]. Disponível em: http://www.ohri.ca/programs/clinical_epidemiology/ oxford.asp.

21. Moher D, Shamseer L, Clarke M, Ghersi D, Liberati A, Petticrew M, Shekelle P, Stewart LA, PRISMA-P Group. Preferred reporting items for systematic review and meta-analysis protocols (PRISMA-P) 2015 statement. Syst Rev 2015; 4(1):1.

22. Chukwulebe SB, Kim HS, McCarthy DM, Courtney DM, Lank PM, Gravenor SJ, Dresden SM. Potentially Inappropriate Medication Prescriptions for Older Adults with Painful Conditions and Association with Return Emergency Department Visits. J Am Geriatr Soc 2019; 67(4):719-725.

23. Lester E, Dykstra M, Grant C, Fawcett V, Tsang B, Widder S. High-risk medications in older patients with trauma: a cross-sectional study of risk mitigation. Can J Surg 2019; 62(2):100-104.

24. Ma Z, Zhang C, Cui X, Liu L. Comparison of three criteria for potentially inappropriate medications in Chinese older adults. Clin Interv Aging 2019; 14:65-72.

25. Chang CB, Lai HY, Hwang SJ, Yang SY, Wu RS, Liu HC, Chan DC. Prescription of potentially inappropriate medication to older patients presenting to the emergency department: a nationally representative population study. Sci Rep 2018; 8(1):11727.

26. Komagamine J. Prevalence of potentially inappropriate medications at admission and discharge among hospitalised elderly patients with acute medical illness at a single centre in Japan: a retrospective cross-sectional study. BMJ Open 2018; 8(7):e021152.

27. Komagamine J, Sugawara K, Hagane K. Characteristics of elderly patients with polypharmacy who refuse to participate in an in-hospital deprescribing intervention: a retrospective cross-sectional study. $B M C$ Geriatrics 2018; 18(1):1-6.
28. Najjar MF, Sulaiman SAS, Al Jeraisy M, Balubaid H. The impact of a combined intervention program: an educational and clinical pharmacist's intervention to improve prescribing pattern in hospitalized geriatric patients at King Abdulaziz Medical City in Riyadh, Saudi Arabia. Ther Clin Risk Manag 2018; 14:557-564.

29. Sarwar MR, Dar AR, Mahar SY, Riaz T, Danish U, Iftikhar S. Assessment of prescribing potentially inappropriate medications listed in Beers criteria and its association with the unplanned hospitalization: a cross-sectional study in Lahore, Pakistan. Clin Interv Aging 2018; 13:1485-1495.

30. Sharma J, Parulekar M, Stewart P, Blatt M, Zielonka T, Nyirenda T, Rogers C, Tank L. Geriatric Consultation Reduces High-risk Medication Usage at Discharge in Elderly Trauma Patients. Cureus 2018; 10(11):e3649.

31. Komagamine J, Hagane K. Intervention to improve the appropriate use of polypharmacy for older patients with hip fractures: an observational study. $B M C$ Geriatrics 2017; 17(1):288.

32. Li H, Pu S, Liu Q, Huang X, Kuang J, Chen L, Shen J, Cheng S, Wu T, Li R, Li Y, Mo L, Jiang W, Song Y, $\mathrm{He}$ J. Potentially inappropriate medications in Chinese older adults: The beers criteria compared with the screening tool of older persons' prescriptions criteria. Geriatr Gerontol Int 2017; 17(11):1951-1958.

33. Zhang X, Zhou S, Pan K, Li X, Zhao X, Zhou Y, Cui Y, Liu X. Potentially inappropriate medications in hospitalized older patients: a cross-sectional study using the Beers 2015 criteria versus the 2012 criteria. Clin Interv Aging 2017; 12:1697-1703.

34. Arellano C, Saldivia G, Cordova P, Fernandez P, Morales F, Lopez M, Villa L. Using two tools to identify Potentially Inappropriate Medications (PIM) in elderly patients in Southern Chile. Arch Gerontol Geriatr 2016; 67:139-144.

35. Di Giorgio C, Provenzani A, Polidori P. Potentially inappropriate drug prescribing in elderly hospitalized patients: an analysis and comparison of explicit criteria. Int J Clin Pharm 2016; 38(2):462-468.

36. Rongen S, Kramers C, O'Mahony D, Feuth TB, Olde Rikkert MG, Ahmed AI. Potentially inappropriate prescribing in older patients admitted to psychiatric hospital. Int J Geriatr Psychiatry 2016; 31(2):137-145.

37. Yang PJ, Lee YT, Tzeng SL, Lee HC, Tsai CF, Chen CC, Chen SC, Lee MC. Potentially Inappropriate Prescribing in Disabled Older Patients with Chronic Diseases: A Screening Tool of Older Persons' Potentially Inappropriate Prescriptions versus Beers 2012 Criteria. Med Princ Pract 2015; 24(6):565-570.

38. Matanovic SM, Vlahovic-Palcevski V. Potentially inappropriate prescribing to the elderly: comparison of new protocol to Beers criteria with relation to hospitalizations for ADRs. Eur J Clin Pharmacol 2014; 70(4):483-490.

39. Tosato M, Landi F, Martone AM, Cherubini A, Corsonello A, Volpato S, et al. Potentially inappropriate drug use among hospitalised older adults: results from the CRIME study. Age Ageing 2014; 43(6):767-773.

40. Napolitano F, Izzo MT, Di Giuseppe G, Angelillo IF. Frequency of inappropriate medication prescription in hospitalized elderly patients in Italy. PloS One 2013; 8(12):e82359. 
41. Praxedes MFS, Telles Filho PCP, Pinheiro MLP. Identificação e análise de prescrições de medicamentos potencialmente inapropriados para idosos em uma instituição hospitalar. Cien Cuid Saude 2011; 10(2):338-344.

42. Sakuma M, Morimoto T, Matsui K, Seki S, Kuramoto N, Toshiro J, Murakami J, Fukui T, Saito M, Hiraide A, Bates DW. Epidemiology of potentially inappropriate medication use in elderly patients in Japanese acute care hospitals. Pharmacoepidemiology Drug Saf 2011; 20(4):386-392.

43. Zhou B, Huang Y, Li H, Sun W, Liu J. Proton-pump inhibitors and risk of fractures: an update meta-analysis. Osteoporos Int 2016; 27(1):339-347.

44. Deshpande A, Pant C, Pasupuleti V, Rolston DD, Jain A, Deshpande N, Thota P, Sferra TJ, Hernandez AV. Association between proton pump inhibitor therapy and Clostridium difficile infection in a meta-analysis. Clin Gastroenterol Hepatol 2012; 10(3):225-233.

45. Giuliano C, Wilhelm SM, Kale-Pradhan PB. Are proton pump inhibitors associated with the development of community-acquired pneumonia? A meta-analysis. Expert Rev Clin Pharmacol 2012; 5(3):337-344.

46. Pandya U, O'Mara MS, Wilson W, Opalek J, Lieber M. Impact of preexisting opioid use on injury mechanism, type, and outcome. J Surg Res 2015; 198(1):7-12.

47. Daoust R, Paquet J, Moore L, Emond M, Gosselin S, Lavigne $\mathrm{G}$, Choinière $\mathrm{M}$, Boulanger $\mathrm{A}$, Mac-Thiong J-M, Chauny J-M. Recent opioid use and fall-related injury among older patients with trauma. CMAJ 2018; 190(16):E500-E506.

48. Pickering G. Analgesic use in the older person. Curr Opin Support Palliat Care 2012; 6(2):207-212.

49. Scheiman JM. Prevention of NSAID-Induced Ulcers. Curr Treat Options Gastroenterol 2008; 11(2):125-134.

50. Trelle S, Reichenbach S, Wandel S, Hildebrand P, Tschannen B, Villiger PM, Egger M, Jüni P. Cardiovascular safety of non-steroidal anti-inflammatory drugs: network meta-analysis. BMJ 2011; 342:c7086.

51. American Geriatrics Society Panel on Pharmacological Management of Persistent Pain in Older Persons. Pharmacological management of persistent pain in older persons. J Am Geriatr Soc 2009; 57(8):13311346.

52. Bakken MS, Engeland A, Engesaeter LB, Ranhoff AH, Hunskaar S, Ruths S. Risk of hip fracture among older people using anxiolytic and hypnotic drugs: a nationwide prospective cohort study. Eur J Clin Pharmacol 2014; 70(7):873-880.

53. Steinman MA, Beizer JL, DuBeau CE, Laird RD, Lundebjerg NE, Mulhausen P. How to Use the American Geriatrics Society 2015 Beers Criteria-A Guide for Patients, Clinicians, Health Systems, and Payors. J Am Geriatr Soc 2015; 63(12):e1-e7.

54. Fried TR, O'Leary J, Towle V, Goldstein MK, Trentalange M, Martin DK. Health outcomes associated with polypharmacy in community-dwelling older adults: a systematic review. J Am Geriatr Soc 2014; 62(12):2261-2272

55. Williams S, Miller G, Khoury R, Grossberg GT. Rational deprescribing in the elderly. Ann Clin Psychiatry 2019; 31(2):144-152.
56. Bala SS, Chen TF, Nishtala PS. Reducing Potentially Inappropriate Medications in Older Adults: A Way Forward. Can J Aging 2019; 38(4):419-433.

57. Scott IA, Hilmer SN, Reeve E, Potter K, Le Couteur D, Rigby D, Gnjidic D, Del Mar CB, Roughead EE, Page A, Jansen J, Martin JH. Reducing inappropriate polypharmacy: the process of deprescribing. JAMA Intern Med 2015; 175(5):827-834.

58. Iankowitz $\mathrm{N}$, Dowden $\mathrm{M}$, Palomino S, Uzokwe $\mathrm{H}$, Worral P. The effectiveness of computer system tools on potentially inappropriate medications ordered at discharge for adults older than 65 years of age: a systematic review. JBI Libr Syst Rev 2012; 10(13):798-831.

59. Drenth-van Maanen AC, Leendertse AJ, Jansen PAF, Knol W, Keijsers C, Meulendijk MC, van Marum RJ. The Systematic Tool to Reduce Inappropriate Prescribing (STRIP): Combining implicit and explicit prescribing tools to improve appropriate prescribing. J Eval Clin Pract 2018; 24(2):317-322.

Artigo apresentado em 01/07/2019

Aprovado em 01/06/2020

Versão final apresentada em 03/06/2020

Editores-chefes: Romeu Gomes, Antônio Augusto Moura da Silva 
